\title{
A Universal Low-Complexity Symbol-to-Bit Soft Demapper
}

\author{
Qi Wang, Qiuliang Xie, Zhaocheng Wang, Senior Member, IEEE, Sheng Chen, Fellow, IEEE, and \\ Lajos Hanzo, Fellow, IEEE
}

\begin{abstract}
High-order constellations are commonly used for achieving high bandwidth efficiency in most communication systems. However, the complexity of the multiplication operations associated with the standard max-sum approximation of the maximum a posteriori probability in the log-domain (Max-Log-MAP) symbol-to-bit demapper is very high. In this contribution, we conceive a low-complexity universal soft demapper, which reduces the demapper's complexity considerably for the binary-reflected Gray-labeled pulse amplitude modulation (PAM), phase shift keying (PSK), quadrature amplitude modulation (QAM), and amplitude phase-shift keying (APSK) relying on product constellation labeling (product-APSK). Our theoretical analysis demonstrates that the proposed demapper has exactly the same performance as the Max-Log-MAP demapper for the Gray-labeled PAM, PSK, and QAM. Our theoretical analysis and simulation results also demonstrate that for the Gray-labeled product-APSK, the performance degradation of the proposed simplified soft demapper is negligible for both 64-ary and 256-ary constellations compared with the Max-Log-MAP demapper.
\end{abstract}

Index Terms-Amplitude phase-shift keying (APSK), Max-Log-MAP, phase-shift keying (PSK), pulse amplitude modulation (PAM), quadrature amplitude modulation (QAM), soft demapper.

\section{INTRODUCTION}

H IGH-ORDER constellations are preferred in many transmission systems, as they are capable of achieving high bandwidth efficiency. For example, 256-ary quadrature amplitude modulation (256QAM) and 4096QAM are employed by the second-generation digital terrestrial television broadcasting standard (DVB-T2) [1] and the second-generation digital cable television broadcasting standard (DVB-C2) [2], respectively.

Manuscript received March 28, 2013; revised June 14, 2013; accepted June 30, 2013. Date of publication August 21, 2013; date of current version January 13, 2014. This work was supported in part by the National Natural Science Foundation of China under Grant 61271266, by the National Key Basic Research Program of China under Grant 2013CB329203, and by the National High Technology Research and Development Program of China under Grant 2012AA011704. The review of this paper was coordinated by Prof. W. A. Hamouda.

Q. Wang and Z. Wang are with the Tsinghua National Laboratory for Information Science and Technology, Department of Electronic Engineering, Tsinghua University, Beijing 100084, China (e-mail: qiwang11@mails. tsinghua.edu.cn; zcwang@ mail.tsinghua.edu.cn).

Q. Xie is with the Department of Radiation Oncology, University of California, Los Angeles, CA 90024 USA (e-mail: xieqiuliang@gmail.com).

$\mathrm{S}$. Chen is with Electronics and Computer Science, University of Southampton, Southampton SO17 1BJ, U.K., and also with King Abdulaziz University, Jeddah 21589, Saudi Arabia (e-mail: sqc@ecs.soton.ac.uk).

L. Hanzo is with Electronics and Computer Science, University of Southampton, Southampton SO17 1BJ, U.K. (e-mail: 1h@ecs.soton.ac.uk).

Color versions of one or more of the figures in this paper are available online at http://ieeexplore.ieee.org.

Digital Object Identifier 10.1109/TVT.2013.2272640
Furthermore, 128QAM is recommended by the long-term evolution advanced (LTE-Advanced) standards [3], which supports reception even for high-velocity vehicular communications. However, for these high-order modulation schemes, a highcomplexity symbol-to-bit demapper is required when using the conventional maximum a posteriori probability based in the log-domain (Log-MAP) demapping algorithm [4]. Albeit the max-sum-approximation-based version of the Log-MAP (Max-Log-MAP) demapper [5] eliminates the high-complexity exponential and logarithmic operations in the Log-MAP algorithm, the number of multiplications remains high, and the complexity of the Max-Log-MAP algorithm is on the order of $\mathrm{O}\left(2^{m}\right)$, where $2^{m}$ denotes the constellation size with $m$ representing the number of bits per symbol.

Numerous simplified demapper algorithms have been proposed for specific constellations. In [6], a bit-metric-generation approach is proposed for phase-shift keying (PSK) using Gray labeling, which recursively generates bit metrics based on a simplified function. This recursive demapper achieves the same performance as the Max-Log-MAP demapper, while reducing the number of multiplications by $59 \%$ for 32PSK. By decomposing the $2^{m}$-ary QAM constellation into two independent (inphase and quadrature) $2^{m / 2}$-ary pulse amplitude modulation (PAM) constellations, the complexity of the associated MaxLog-MAP demapper is reduced from $\mathrm{O}\left(2^{m}\right)$ to $\mathrm{O}\left(2^{m / 2}\right)$ [7], [8]. The complexity of the QAM demapper can be further reduced to the order of $\mathrm{O}(m)$ by invoking a piecewise linear approximation, but this inevitably imposes performance degradation [9]. A similar soft demapper is proposed for amplitude PSK (APSK) in [10], where the constellation is partitioned with the aid of simplified hard-decision threshold (HDT)-based boundary lines, and soft information is calculated as the distances between the received signal and the HDT lines. This approximate demapper reduces the number of multiplications to 4 and 11 for 16APSK and 32APSK, respectively. A simplified demapper is also proposed for multilevel coding followed by multistage decoding, which focuses on the APSK signal [11], and the complexity of this APSK demapper is reduced to a constant (neglecting comparison operations) at the cost of exponentially increasing the memory required and necessitating an additional division [11]. In [12], the complexity of the demapper is reduced by reusing the multipliers, and only 16 multipliers are used for all the four modulation modes (QPSK, 8PSK, 16APSK, and 32APSK) in the second-generation digital video broadcasting over satellite (DVB-S2) system. For the constellation rotation and cyclic $Q$ delay modulation of DVB-T2, several simplified demappers are proposed for reducing 
complexity by decreasing the number of the constellation points required for calculating the minimum squared distances [13][15]. For APSK using product constellation labeling (productAPSK), it is shown [16] that a $\left(2^{m_{1}} \times 2^{m_{2}}=2^{m}\right)$-ary APSK constellation can be regarded as the product of $2^{m_{1}}$-ary PSK and pseudo $2^{m_{2}}$-ary PAM, and a simplified demapper is proposed in [17], which reduces the complexity of the demapper from $\mathrm{O}\left(2^{m}\right)$ to $\mathrm{O}\left(2^{m_{1}}\right)+\mathrm{O}\left(2^{m_{2}}\right)$.

All the previously mentioned Gray labeling functions designed for the various constellations are the classic binaryreflected Gray labeling schemes proposed by Gray in 1953 as a means of reducing the number of bit errors, where two adjacent constellation points differ in only one bit [18]. In [19], Agrell et al. showed that the binary-reflected Gray labeling is the optimal labeling for PAM, PSK, and QAM, which achieves the lowest possible bit error probability among all possible labeling functions for the additive white Gaussian noise (AWGN) channel.

Against this background, in this contribution, a universal low-complexity soft demapper is proposed for various binary-reflected Gray-labeled constellations. By exploiting the symmetry of Gray-labeled constellations, we show that the complexity of a $2^{m}$-ary demapper can be reduced from $\mathrm{O}\left(2^{m}\right)$ to $\mathrm{O}(m)$. Moreover, our proposed low-complexity soft demapper attains the same performance as the Max-Log-MAP demapper for PAM, PSK, and QAM, whereas the performance degradation of our low-complexity soft demapper is negligible for product-APSK, in comparison with the Max-Log-MAP solution.

The rest of this paper is organized as follows. In Section II, the standard Max-Log-MAP demapper is highlighted. In Section III, our simplified soft demapper is proposed, and its performance and complexity are analyzed in detail. In Section IV, the performance of both the proposed lowcomplexity demapper and the conventional Max-Log-MAP demapper is quantified for Gray-labeled QAM and productAPSK for transmission over both AWGN and Rayleigh fading channels. Our conclusions are drawn in Section V.

The following notations are employed throughout this contribution. Uppercase calligraphic letters denote sets, e.g., $\mathcal{X}$. Boldface lowercase letters represent vectors, e.g., b, whose $i$ th element is written as $b_{i}$. Uppercase letters denote random variables (RVs), e.g., $X$, whereas the corresponding lowercase letters represent their realizations, e.g., $x . P(x)$ is used for the probability mass function (pmf) of a discrete $\mathrm{RV} X$, and $p(x)$ denotes the probability density function (pdf) of a continuous RV X.P $P(y \mid x)$ represents the conditional pmf of $Y=y$ given $X=x$, whereas $p(y \mid x)$ represents the conditional pdf of $Y=y$ given $X=x$. The magnitude operator is denoted by $|\cdot|$.

\section{System Model With MaX-Log-MaXimum A POSTERIORI Demapper}

At the transmitter of a coded system, the coded bits are grouped into bit vectors, each with the length of $m$ and denoted by $\mathbf{b}=\left(\begin{array}{llll}b_{0} & b_{1} & \ldots & b_{m-1}\end{array}\right)$. Bit vector $\mathbf{b}$ is then mapped onto constellation point $x \in \mathcal{X}$ for transmission, where $\mathcal{X}=$ $\left\{x_{k}, 0 \leq k<2^{m}\right\}$ denotes the signal set of size $2^{m}$.
At the receiver, the soft information for each coded bit is calculated based on received signal $y$, which is then passed to the decoder. For the Log-MAP demapper, the soft information on the $i$ th bit is expressed in the form of the log-likelihood ratio (LLR) $L_{i}$ according to [17]

$$
\begin{aligned}
L_{i} & =\log \frac{P\left(b_{i}=0 \mid y\right)}{P\left(b_{i}=1 \mid y\right)}=\log \frac{\sum_{x \in \mathcal{X}_{i}^{(0)}} P(x \mid y)}{\sum_{x \in \mathcal{X}_{i}^{(1)}} P(x \mid y)} \\
& =\log \frac{\sum_{x \in \mathcal{X}_{i}^{(0)}} p(y \mid x)}{\sum_{x \in \mathcal{X}_{i}^{(1)}} p(y \mid x)}
\end{aligned}
$$

for $0 \leq i<m$, where $\mathcal{X}_{i}^{(b)}$ denotes the signal subset of $\mathcal{X}$ with the $i$ th bit being $b \in\{0,1\}$. The last equality in (1) follows from Bayes' rule and the assumption that signals $x_{k}, 0 \leq k<2^{m}$ are equiprobable.

A flat-fading channel is modeled as $y=h x+n$, where $h$ denotes the complex-valued channel state information (CSI), and $n$ stands for the complex-valued AWGN with zero mean and variance $N_{0} / 2$ per dimension. When the perfect CSI $h$ is available at the receiver, the conditional pdf $p(y \mid x)$ in (1) can be written as $p(y \mid x)=\left(1 / \pi N_{0}\right) \exp \left(-|y-h x|^{2} / N_{0}\right)$. Observe that given the availability of perfect CSI, the received signal can be phase equalized, after which only the amplitude of CSI $h$ is required. Thus, we simply assume that $h$ is nonnegative real valued. By using the well-known max-sum approximation of $\sum_{j} z_{j} \approx \max _{j} z_{j}$ for nonnegative $z_{j}$, where the summation is dominated by the largest term, the conventional Max-LogMAP demapper is readily formulated as

$$
\begin{aligned}
L_{i} & \approx \log \frac{\max _{x \in \mathcal{X}_{i}^{(0)}} p(y \mid x)}{\max _{x \in \mathcal{X}_{i}^{(1)}} p(y \mid x)} \\
& =-\frac{1}{N_{0}}\left(\min _{x \in \mathcal{X}_{i}^{(0)}}|y-h x|^{2}-\min _{x \in \mathcal{X}_{i}^{(1)}}|y-h x|^{2}\right) .
\end{aligned}
$$

The Max-Log-MAP of (2) is a fairly accurate approximation of the Log-MAP of (1) in the high signal-to-noise ratio (SNR) region, and it avoids the complex exponential and logarithmic operations. For each received signal, the Max-Log-MAP demapper calculates all the $2^{m}$ squared Euclidean distances, i.e., $|y-h x|^{2}$ for every $x \in \mathcal{X}$, to find the two minimum terms described in (2). Therefore, its complexity quantified in terms of multiplications is on the order of $\mathrm{O}\left(2^{m}\right)$.

\section{Proposed Simplified Soft Demapper}

After carefully examining (2), it is interesting to note that item $\min _{x \in \mathcal{X}}|y-h x|^{2}$, i.e., the squared Euclidean distance from received signal $y$ to the nearest constellation point $x^{*}$, always appears in (2), and it is equal to either $\min _{x \in \mathcal{X}_{i}^{(0)}} \mid y-$ $\left.h x\right|^{2}$ or $\min _{x \in \mathcal{X}_{i}^{(1)}}|y-h x|^{2}$, depending on the $i$ th bit of $x^{*}$ being 0 or 1 . In other words, $\left|y-h x^{*}\right|^{2}$ is always one of the two terms in (2). By denoting the bit vector that maps to signal $x^{*}$ as $\mathbf{b}^{*}=\left(b_{0}^{*} b_{1}^{*} \ldots b_{m-1}^{*}\right)$, the other item in (2) represents the squared Euclidean distance from $y$ to the nearest constellation 
point in subset $\mathcal{X}_{i}^{\left(\overline{b_{i}^{*}}\right)}$, which is denoted by $x_{i, \overline{b_{i}^{*}}}^{*}$, where we have $\bar{b}=1-b$.

For Gray-labeled constellations, we will show that $x^{*}$ and $x_{i, b_{i}^{*}}^{*}, 0 \leq i<m$, can be determined by using simple comparison and addition operations. Afterward, we only have to calculate the $m+1$ squared Euclidean distances, i.e., $\left|y-h x^{*}\right|^{2}$ and $\left|y-h x_{i, \bar{b}}^{*}\right|^{2}$ for $0 \leq i<m$. Therefore, the complexity of our proposed demapper is on the order of $\mathrm{O}(\mathrm{m})$.

Accordingly, we divide the demapping procedure into three steps: 1) finding $x^{*}$ and $\mathbf{b}^{*}$; 2) determining $x_{i, \overline{b_{i}^{*}}}^{*}$; and 3) calculating $L_{i}$ according to (2). For binary-reflected Graylabeled constellations, we have the following lemma from [20], describing how to obtain $\mathbf{b}^{*}$.

Lemma 1: For binary-reflected Gray labeling $\mathbf{b} \rightarrow x_{k}$, by denoting $\mathbf{c}^{k}=\left(\begin{array}{llll}c_{0}^{k} & c_{1}^{k} & \ldots & c_{m-1}^{k}\end{array}\right)$ as the binary representation of index $k$ with the least significant bit (LSB) as the rightmost bit, $\mathbf{b}$ can be calculated as

$$
\mathbf{b}=\left(c_{0}^{k} c_{1}^{k} \ldots c_{m-1}^{k}\right) \oplus\left(\begin{array}{llll}
0 & c_{0}^{k} \ldots c_{m-2}^{k}
\end{array}\right)
$$

where $\oplus$ represents the bitwise XOR operation.

The expressions generated for determining $x^{*}$ and $x_{i, \overline{b_{i}^{*}}}^{*}$ are slightly different for various constellations. In the following, the simplified soft demappers designed for the Gray-labeled PAM, QAM, PSK, and product-APSK are presented in detail.

\section{A. PAM Demapper}

Without loss of generality, we assume that all the signals associated with PAM are real valued. For the $2^{m}$-ary Gray-labeled PAM, we denote the constellation points as $x_{0}, x_{1}, \ldots, x_{2^{m}-1}$ with the $k$ th constellation point $x_{k}$ given by $x_{k}=\delta\left(-\left(2^{m}-\right.\right.$ 1) $+2 k) / 2$, where $\delta$ denotes the distance between each pair of adjacent constellation points. The detailed PAM demapping procedure is given as follows.

1) Find $x^{*}$ and $\mathbf{b}^{*}$. For $2^{m}$-PAM, signal space can be divided into $2^{m}$ intervals separated by amplitude thresholds $-\left(2^{m-1}-1\right) \delta,-\left(2^{m-1}-2\right) \delta, \ldots,\left(2^{m-1}-1\right) \delta$. Multiplying $h$ with the thresholds can be implemented by SHIFT-ADD operations, since the thresholds are constants. Additionally, we can use the binary-search algorithm to find the specific interval in which $y$ is located. Therefore, only $m$ comparison operations are required for obtaining $x^{*}=x_{k^{*}}$. The corresponding bit vector $\mathbf{b}^{*}$ can then be calculated according to Lemma 1. An example for the Gray-labeled 8PAM (Gray-8PAM) constellation is shown in Fig. 1, where we have $k^{*}=2$ and $\mathbf{b}^{*}=\left(\begin{array}{lll}0 & 1 & 1\end{array}\right)$.

2) Determine $x_{i, \bar{b}_{*}^{*}}^{*}$. Considering the symmetric structure of Gray-labeled PAM constellations, we have the following lemma for computing $x_{i, \overline{b_{i}^{*}}}^{*}$, which only requires the binary representation of $k^{*}$ and addition operations, instead of the need to calculate all the squared Euclidean distances from $y$ to the constellation points in subset $\mathcal{X}_{i}^{\left(\overline{b_{i}^{*}}\right)}$ and compare all the resultant $2^{m-1}$ metrics.

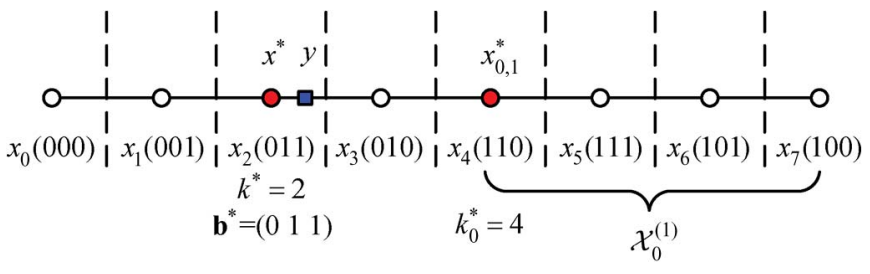

Fig. 1. Gray-8PAM constellation and illustration of demapping for the 0th bit over the AWGN channel.

Lemma 2: For the binary-reflected Gray PAM $\mathbf{b}^{*} \rightarrow x_{k^{*}}$, where $x_{k^{*}}$ is the nearest constellation point to received signal $y$, let $\mathbf{c}^{k^{*}}=\left(\begin{array}{lll}c_{0}^{*} & c_{1}^{k^{*}} \ldots & c_{m-1}^{k^{*}}\end{array}\right)$ be the binary representation of $k^{*}$ with the LSB as the rightmost bit. Then, the nearest constellation point to $y$ in subset $\mathcal{X}_{i}^{\left(\overline{b_{i}^{*}}\right)}$, namely, $x_{i, \overline{b_{i}^{*}}}^{*}$, can be determined according to

$$
x_{i, \overline{b_{i}^{*}}}^{*}=x_{k_{i}^{*}}
$$

where

$$
k_{i}^{*}=2^{m-i-1}-c_{i}^{k^{*}}+\sum_{j=0}^{i-1} c_{j}^{k^{*}} 2^{m-j-1} .
$$

Proof: See Appendix A.

Calculate $L_{i}$ according to (2). After obtaining $x^{*}, \mathbf{b}^{*}$, and $x_{i, \overline{b_{i}^{*}}}^{*}$, we can rewrite $L_{i}$ as

$$
L_{i}=-\frac{1}{N_{0}}\left(1-2 b_{i}^{*}\right)\left(\left|y-h x^{*}\right|^{2}-\left|y-h x_{i, \overline{b_{i}^{*}}}^{*}\right|^{2}\right) .
$$

It is clear that (6) is equivalent to (2) for the Gray-labeled PAM. Hence, the performance of the proposed simplified soft demapper is exactly the same as that of the standard Max-LogMAP demapper, while its complexity is reduced from $\mathrm{O}\left(2^{m}\right)$ to $\mathrm{O}(m)$.

\section{B. QAM Demapper}

The $2^{m}$-ary square Gray-labeled QAM can be decomposed into two independent (in-phase and quadrature phase) $2^{m / 2}$-ary Gray-labeled PAMs, and we can apply our proposed simplified PAM demapper to each of these two Gray-labeled PAMs. Thus, the complexity of our simplified Gray-labeled QAM demapper is reduced from $\mathrm{O}\left(2^{m}\right)$ to $\mathrm{O}(\mathrm{m})$ without suffering any performance loss, in comparison to the standard Max-Log-MAP demapper.

\section{PSK Demapper}

By applying the same idea to PSK demapping, we can also reduce the complexity from $\mathrm{O}\left(2^{m}\right)$ to $\mathrm{O}(\mathrm{m})$ without any performance loss, compared with the Max-Log-MAP solution. For $2^{m}$-ary Gray-labeled PSK, the signal set can be written in the polar coordinate format as $\mathcal{X}=\left\{x_{k}=\sqrt{E_{s}} \exp (\mathrm{j}(2 k+\right.$ 1) $\left.\left.\pi / 2^{m}\right), 0 \leq k<2^{m}\right\}$, where $E_{s}$ denotes the energy of the transmitted signals, and $\mathrm{j}=\sqrt{-1}$. An example of the Gray8PSK constellation is shown in Fig. 2. 


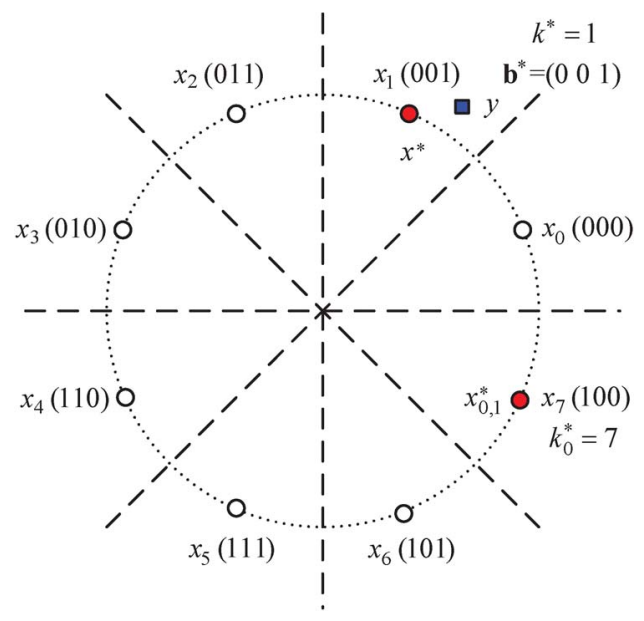

Fig. 2. Gray-8PSK constellation and illustration of demapping for the zeroth bit over the AWGN channel.

Let us express the phase-equalized received signal $y$ in the polar coordinate format as $y=\rho_{y} \exp \left(\mathrm{j} \varphi_{y}\right)$, where $\rho_{y}$ and $\varphi_{y}$ denote the amplitude and phase of $y$, respectively, and $0 \leq$ $\varphi_{y}<2 \pi$. Then, the squared Euclidean distance $|y-h x|^{2}$ can be written as

$$
\begin{aligned}
|y-h x|^{2} & =\left|\rho_{y} \exp \left(\mathrm{j} \varphi_{y}\right)-h \sqrt{E_{s}} \exp \left(\mathrm{j} \varphi_{x}\right)\right|^{2} \\
& =\rho_{y}^{2}+h^{2} E_{s}-2 \rho_{y} h \sqrt{E_{s}} \cos \left(\varphi_{x}-\varphi_{y}\right) \\
& =\rho_{y}^{2}+h^{2} E_{s}-2 \rho_{y} h \sqrt{E_{s}} \cos (\phi(x, y))
\end{aligned}
$$

where $\varphi_{x}$ is the phase of $x$, and $\phi(x, y)$ is defined as

$$
\phi(x, y)=\left\{\begin{array}{ll}
\left|\varphi_{x}-\varphi_{y}\right|, & 0 \leq\left|\varphi_{x}-\varphi_{y}\right| \leq \pi \\
2 \pi-\left|\varphi_{x}-\varphi_{y}\right|, & \pi<\left|\varphi_{x}-\varphi_{y}\right|<2 \pi
\end{array} .\right.
$$

It is obvious that $\phi(x, y) \in[0, \pi]$ and is commutative, i.e., $\phi(x, y)=\phi(y, x)$. The mapping defined in (8) also satisfies the triangle inequality, that is, $\forall x, y, z \in \mathbb{C}$, we have

$$
\phi(x, z) \leqslant \phi(x, y)+\phi(y, z)
$$

where $\mathbb{C}$ denotes the complex-valued space. The proof is given in Appendix B. Therefore, $\phi(x, y)$ defines a distance over $\mathbb{C}$, which is referred to as the phase distance of $x$ and $y$ in this paper.

Furthermore, multiplying $x \in \mathbb{C}$ with a positive value does not change the phase of $x$, i.e., $\varphi_{h x}=\varphi_{x}, \forall h>0$. Hence, we have $\phi(h x, y)=\phi(x, y), \forall h>0$. Since the cosine function is a decreasing function in $[0, \pi]$, minimizing the squared Euclidean distance $|y-h x|^{2}$ of (7) is equivalent to minimizing phase distance $\phi(x, y)$. Therefore, we can simply use the phase of the signal in the search process of the PSK demapper, and the resultant PSK demapping procedure is detailed as follows.

1) Find $x^{*}$ and $\mathbf{b}^{*}$. The signal space of the $2^{m}$-ary Graylabeled PSK can be divided into $2^{m}$ phase intervals separated by phase thresholds $0, \pi / 2^{m-1}, \ldots,\left(2^{m}-\right.$ 1) $\pi / 2^{m-1}$, as shown in Fig. 2. Signal $x^{*}$ can be obtained by comparing $\varphi_{y}$ with the phase thresholds, which only needs $m$ comparisons using the binary-search algorithm.
Similar to the PAM demapper, after finding $x^{*}=x_{k^{*}}$, the corresponding bit vector $\mathbf{b}^{*}$ is calculated according to Lemma 1. For the case shown in Fig. 2, we have $k^{*}=1$ and $\mathbf{b}^{*}=\left(\begin{array}{lll}0 & 0 & 1\end{array}\right)$.

2) Determine $x_{i, \overline{b_{i}^{*}}}^{*}$. Unlike the PAM constellation, the PSK constellation is circularly symmetric, and the phase distance function we used for comparisons is defined in a piecewise fashion. Therefore, calculating $x_{i, \overline{b_{i}^{*}}}^{*}$ for the PSK demapper is slightly different from that of the PAM demapper. We have the following lemma for computing $x_{i, \overline{b_{i}^{*}}}^{*}$ of Gray-labeled PSK.

Lemma 3: For the binary-reflected Gray PSK $\mathbf{b}^{*} \rightarrow x_{k^{*}}$, where $x_{k^{*}}$ is the constellation point nearest to received signal $y$, let $\mathbf{c}^{k^{*}}=\left(\begin{array}{lll}c_{0}^{k^{*}} & c_{1}^{k^{*}} \ldots & c_{m-1}^{k^{*}}\end{array}\right)$ be the binary representation of $k^{*}$ with the LSB as the rightmost bit. Then, the point nearest to $y$ in subset $\mathcal{X}_{i}^{\left(\overline{b_{i}^{*}}\right)}$, namely, $x_{i, \overline{b_{i}^{*}}}^{*}$, can be determined according to

$$
x_{i, \overline{b_{i}^{*}}}^{*}=x_{k_{i}^{*}}
$$

where

$$
k_{i}^{*}=\left\{\begin{array}{ll}
\overline{c_{0}^{k *}} 2^{m-1}+\overline{c_{1}^{k^{*}}}\left(2^{m-1}-1\right), & i=0 \\
2^{m-i-1}-c_{i}^{k^{*}}+\sum_{j=0}^{i-1} c_{j}^{k^{*}} 2^{m-j-1}, & i>0
\end{array} .\right.
$$

Proof: See Appendix C.

3) Calculate $L_{i}$ according to (2). After obtaining $x^{*}, \mathbf{b}^{*}$, and $x_{i, \overline{b_{i}^{*}}}^{*}$, the soft information on the $i$ th bit, i.e., $L_{i}$, is calculated according to (6), which is the same result as that in (2) for the Max-Log-MAP demapper, as is the case for the PAM demapper. Clearly, the performance of this simplified soft demapper is identical to that of the MaxLog-MAP demapper, while only imposing a complexity on the order of $\mathrm{O}(\mathrm{m})$.

\section{Gray-APSK Demapper}

1) Review of Gray-APSK: A generic $M$-ary APSK constellation is composed of $R$ concentric rings, each having uniformly spaced PSK points. More specifically, the $M$-APSK constellation set is given by $\mathcal{X}=\left\{r_{l} \exp \left(\mathrm{j}\left(2 \pi i / n_{l}+\theta_{l}\right)\right), 0 \leq\right.$ $\left.i<n_{l}, 0 \leq l<R\right\}$, in which $n_{l}, r_{l}$, and $\theta_{l}$ denote the number of PSK points, the radius, and the phase shift of the $l$ th ring, respectively, while we have $\sum_{l=0}^{R-1} n_{l}=M$ [21].

In [16], a special APSK constellation was proposed, which consists of $R=2^{m_{2}}$ rings and $n_{l}=2^{m_{1}}$ PSK points on each ring for the $\left(M=2^{m}\right)$-ary APSK, where we have $m_{1}+$ $m_{2}=m$. This kind of APSK is known as the productAPSK and is denoted by $\left(M=2^{m_{1}} \times 2^{m_{2}}\right)$-APSK. The $l$ th radius of the product-APSK constellation, where $0 \leq l<R$, is determined by

$$
r_{l}=\sqrt{-\ln \left(1-(l+1 / 2) 2^{-m_{2}}\right)} .
$$

The $\left(2^{m}=2^{m_{1}} \times 2^{m_{2}}\right)$-APSK can be regarded as the product of $2^{m_{1}}$-ary PSK and $2^{m_{2}}$-ary pseudo PAM, where the 


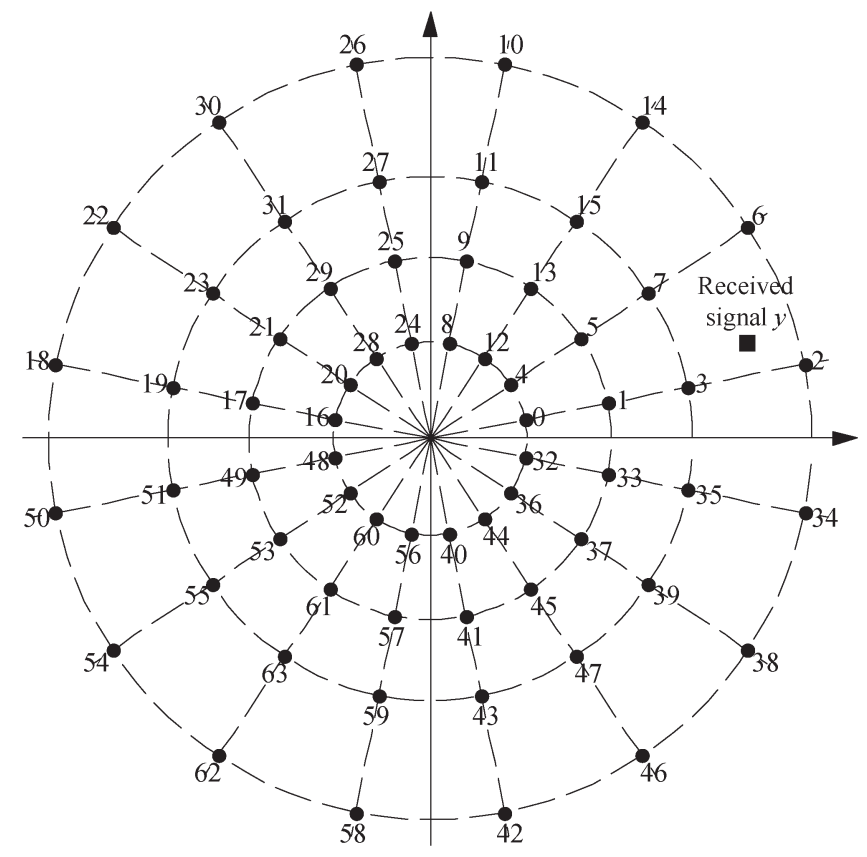

Fig. 3. Gray-labeled $(64=16 \times 4)$-APSK constellation, where the labels are in the decimal form with the binary representation having the LSB as the rightmost bit.

pseudo PAM and PSK sets are given, respectively, by $\mathcal{A}=$ $\left\{r_{l}, 0 \leq l<2^{m_{2}}\right\}$ and $\mathcal{P}=\left\{p_{k}=\exp \left(\mathrm{j} \varphi_{k}\right)\right.$ with $\varphi_{k}=(2 k+$ 1) $\left.\pi / 2^{m_{1}}, 0 \leq k<2^{m_{1}}\right\}$ [17]. We divide the $m$-bit vector $\mathbf{b}$ into two subvectors $\mathbf{b}^{P}$ and $\mathbf{b}^{A}$ of lengths $m_{1}$ and $m_{2}$, respectively. Specifically, $\mathbf{b}^{P}$ consists of the leftmost $m_{1}$ bits of $\mathbf{b}$, whereas $\mathbf{b}^{A}$ contains the rest rightmost $m_{2}$ bits of $\mathbf{b}$. Without loss of generality, $\mathbf{b}^{P}$ is mapped to the equivalent $2^{m_{1}}$ PSK point, and $\mathbf{b}^{A}$ is mapped to the equivalent pseudo $2^{m_{2}}$ PAM point. Gray labeling can be used for mapping the bits to the equivalent constellation signals. This Gray-labeled APSK (Gray-APSK) is a special product-APSK [16], [17]. The Graylabeled $(64=16 \times 4)$-APSK is shown in Fig. 3 .

2) Proposed Demapping Algorithm for Gray-APSK: Like the other constellations previously discussed, the standard MaxLog-MAP demapping designed for Gray-APSK also uses (2). By writing transmitted signal $x$ and received signal $y$ in the polar-coordinate format, the squared Euclidean distance $\mid y-$ $\left.h x\right|^{2}$ for Gray-APSK can be readily expressed as

$$
\begin{aligned}
|y-h x|^{2} & =\rho_{y}^{2}+h^{2} \rho_{x}^{2}-2 h \rho_{x} \rho_{y} \cos (\phi(x, y)) \\
& =\left(\rho_{y} \cos (\phi(x, y))-h \rho_{x}\right)^{2}+\rho_{y}^{2} \sin ^{2}(\phi(x, y))
\end{aligned}
$$

where $\rho_{x}$ and $\rho_{y}$ represent the amplitudes of $x$ and $y$, respectively, and $\phi(x, y)$ is the phase distance between $x$ and $y$, as defined in (8).

Due to the circular symmetry of the Gray-APSK constellation, it is clear that the nearest constellation point $x^{*}$ from $y$ has the smallest phase distance, i.e., $\phi\left(x^{*}, y\right)$ is the smallest one in set $\left\{\phi(x, y), \varphi_{x} \in \mathcal{P}\right\}$, and it is no larger than $\pi / 2^{m_{1}}$, as exemplified in Fig. 3. Furthermore, according to (13), the amplitude of $x^{*}$, which is denoted by $\rho_{x^{*}}$, satisfies

$$
\rho_{x^{*}}=\arg \min _{\rho_{x} \in \mathcal{A}}\left|\rho_{y} \cos \left(\phi\left(x^{*}, y\right)\right)-h \rho_{x}\right| .
$$

After determining the phase and the amplitude of $x^{*}$, it is easy to find the corresponding bit label $\mathbf{b}^{*}$. As for finding $x_{i, \overline{b_{i}^{*}}}^{*}$, this depends on whether the $i$ th bit is related to the phase or the amplitude.

For the bits related to the phase of the Gray-APSK signal, i.e., for $0 \leq i<m_{1}$, the phase of $x_{i, b_{i}^{*}}^{*}$, which is denoted by $\varphi_{x_{i, b_{i}^{*}}^{*}}$, can be readily determined based on Lemma 3 owing to the uniform distribution of the phases, whereas the amplitude of $x_{i, \overline{b_{i}^{*}}}^{*}$, which is denoted by $\rho_{x_{i, \overline{b_{i}^{*}}}^{*}}$, obeys

$$
\rho_{x_{i, \overline{b_{i}^{*}}}^{*}}=\arg \min _{\rho_{x} \in \mathcal{A}}\left|\rho_{y} \cos \left(\phi\left(x_{i, \overline{b_{i}^{*}}}^{*}, y\right)\right)-h \rho_{x}\right| .
$$

For the bits mapped to the amplitude of the Gray-APSK signal, i.e., for $m_{1} \leq i<m$, it is clear that the phase of $x_{i, \overline{b_{i}^{*}}}^{*}$ is exactly the same as that of $x^{*}$, and we may approximately obtain the amplitude of $x_{i, \overline{b_{i}^{*}}}^{*}$ via Lemma 2. However, due to the nonuniformly spaced amplitudes of $\mathcal{A}$, such an approximation may cause some errors, albeit the performance loss is fortunately negligible, as will be detailed later in Section III-D4.

Upon obtaining $x^{*}, \mathbf{b}^{*}$, and $x_{i, b_{i}^{*}}^{*}$, we can readily determine the demapping output of the $i$ th bit based on (6). This simplified Gray-APSK demapping procedure is summarized as follows.

1) Find $x^{*}$ and $\mathbf{b}^{*}$. The phase of $x^{*}$ is determined by minimizing the phase difference from $y$ to $x$ with phase $\varphi_{x} \in \mathcal{P}$, and its amplitude is determined according to (14). Having obtained $\varphi_{x^{*}}=\varphi_{k^{*}}$ and $\rho_{x^{*}}=r_{k^{*}}$, subbit vectors $\mathbf{b}^{P^{*}}$ and $\mathbf{b}^{A^{*}}$ are calculated according to Lemma 1, yielding $\mathbf{b}^{*}=\left(\mathbf{b}^{P^{*}} \mathbf{b}^{A^{*}}\right)$.

2) Determine $x_{i, \bar{b}}^{*}$. For the leftmost $m_{1}$ bits that are related to the phases of the Gray-APSK signals, we can obtain the phase of $x_{i, \overline{b_{i}^{*}}}^{*}$ according to Lemma 3 and its amplitude according to (15). For the rightmost $m_{2}$ bits, i.e., $m_{1} \leq$ $i<m$, the phase of $x_{i, \overline{b^{*}}}^{*}$ is exactly the same as $\varphi_{x^{*}}$, and its amplitude is approximately determined according to Lemma 2.

3) Calculate $L_{i}$ according to (2). After obtaining $x^{*}, \mathbf{b}^{*}$, and $x_{i, \overline{b^{*}}}^{*}$, the soft information on the $i$ th bit, i.e., $L_{i}$, is given by (6), as for the other demappers.

3) Complexity Analysis: Step 1) determines $x^{*}$ and $\mathbf{b}^{*}$. The phase of $x^{*}$ can be readily obtained by simple comparison operations, and its amplitude is determined according to (14), which requires one multiplication for $\rho_{y} \cos \left(\phi\left(x^{*}, y\right)\right)$ and $m_{2}$ comparison operations. Having determined $x^{*}$, calculating $\mathbf{b}^{*}$ only requires some low-complexity XOR operations. The complexity of step 2) is mainly associated with determining the amplitude of $x_{i, \overline{b_{i}^{*}}}^{*}$ according to (15), for $0 \leq i<m_{1}$, which requires one multiplication operation for $\rho_{y} \cos \left(\phi\left(x_{i, \overline{b_{i}^{*}}}^{*}, y\right)\right)$ and $m_{2}$ comparison operations. It is therefore clear that the complexity of the proposed simplified Gray-APSK demapper is $\mathrm{O}\left(2 \times m_{1}+m_{2}\right) \approx \mathrm{O}(m)$, which is dramatically lower than the complexity of $\mathrm{O}\left(2^{m}\right)$ required by the standard Max-LogMAP solution. 


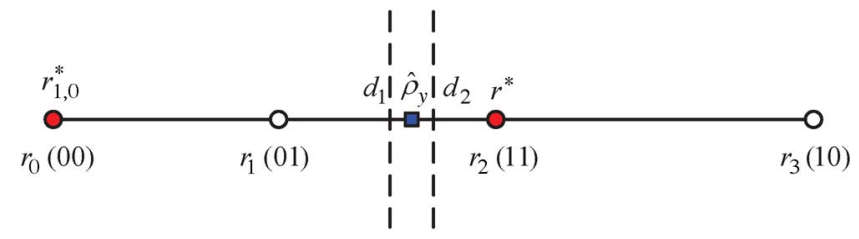

Fig. 4. Pseudo 4 PAM decomposed from the $(64=16 \times 4)$-APSK constellation.

An alternative complexity analysis, which is "easier" to follow is outlined below. The demapper proposed for $\left(2^{m}=\right.$ $2^{m_{1}} \times 2^{m_{2}}$ )-APSK is equivalent to the demapper conceived for $2^{m_{1}}$-ary PSK implemented with the aid of the simplified PSK demapping procedure in Section III-C at the complexity of $\mathrm{O}\left(m_{1}\right)$ and the demapper for the $2^{m_{2}}$-ary pseudo PAM implemented with the aid of the simplified PAM demapping procedure in Section III-A at the complexity of $\mathrm{O}\left(m_{2}\right)$. Therefore, the complexity of the proposed simplified Gray-APSK demapper is approximately $\mathrm{O}\left(m_{1}\right)+\mathrm{O}\left(m_{2}\right) \approx \mathrm{O}(m)$. It is worth emphasizing again that the complexity of our proposed simplified Gray-APSK demapper is also much lower than that of the simplified soft demapper for product-APSK given in [17], which is on the order of $\mathrm{O}\left(2^{m_{1}}\right)+\mathrm{O}\left(2^{m_{2}}\right)$.

4) Performance Analysis: Owing to the fact that the phase of the APSK constellation is uniformly spaced, Lemma 3 always holds when demapping the leftmost $m_{1}$ bits, and the results of the proposed demapper are exactly the same as those of the Max-Log-MAP demapper. However, unlike in the conventional PAM, the distances between pairs of adjacent points in the corresponding pseudo PAM part of the Gray-APSK constellation are not constant, which means that Lemma 2 does not always hold. Therefore, when demapping the rightmost $m_{2}$ bits with the aid of Lemma 2, the resultant $x_{i, \overline{b_{i}^{*}}}^{*}$ may not always be the point nearest to $y$ in subset $\mathcal{X}_{i}^{\left(\overline{b_{i}^{*}}\right)}$, which may slightly increase the absolute value of the LLR in (2) and, consequently, results in some performance degradation. Fortunately, this degradation is negligible. In the following, we present the detailed analysis of this performance loss with the aid of Gray-labeled 64-APSK and 256-APSK.

a) $(64=16 \times 4)$-APSK: As shown in Fig. 4 , to demap the rightmost 2 bits related to the amplitudes in the $(64=16 \times$ 4)-APSK, we have the scalar projection of $y$ in the direction of $\varphi_{x^{*}}$ and the pseudo Gray 4PAM constellation set $\mathcal{A}$. We denote the projection as $\hat{\rho}_{y}=\rho_{y} \cos \left(\phi\left(x^{*}, y\right)\right)$ and the thresholds as $d_{1}=\left(r_{1}+r_{2}\right) / 2$ and $d_{2}=\left(r_{0}+r_{3}\right) / 2$. If $\hat{\rho}_{y}$ is smaller than $d_{1}$, we have $r^{*}=r_{0}$ or $r_{1}$, and the zeroth bit of $\mathbf{b}^{A^{*}}$ must be 0 . The constellation subset with the zeroth bit being 1 is $\mathcal{A}_{0}^{(1)}=\left\{r_{2}, r_{3}\right\}$, and obviously, the nearest point to $\hat{\rho}_{y}$ in $\mathcal{A}_{0}^{(1)}$ is $r_{0,1}^{*}=r_{2}$, which is identical to the result given by Lemma 2 . If $\hat{\rho}_{y}$ is larger than $d_{1}$, we have $b_{0}^{A *}=1$ and $r_{0,0}^{*}=r_{1}$, which is also the same result given by Lemma 2 . Therefore, the proposed demapper achieves the same result as the Max-LogMAP demapper for the zeroth bit of the pseudo 4PAM, and no error is introduced.

However, for the first bit of the pseudo 4PAM, when $\hat{\rho}_{y}$ falls in the interval of $\left(d_{1}, d_{2}\right)$ known as the error interval, ${ }^{1}$

\footnotetext{
${ }^{1}$ Here, we have $d_{1}<d_{2}$ according to (12).
}

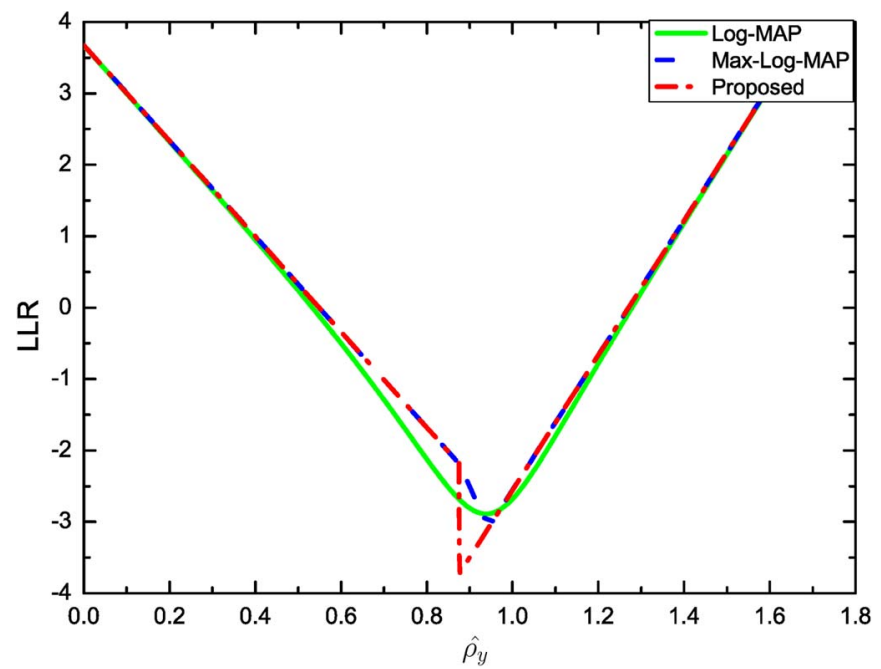

Fig. 5. LLR of the first bit of the pseudo 4PAM decomposed from $(64=16 \times 4)$-APSK over the AWGN channel with $E_{s} / N_{0}=10 \mathrm{~dB}$.

the nearest constellation point to $\hat{\rho}_{y}$ in $\mathcal{A}$ is $r^{*}=r_{2}$, and we have $\mathbf{b}^{A^{*}}=\left(\begin{array}{ll}1 & 1\end{array}\right)$ and $\mathcal{A}_{1}^{(0)}=\left\{r_{0}, r_{3}\right\}$. The point nearest to $\hat{\rho}_{y}$ in $\mathcal{A}_{1}^{(0)}$ is supposed to be $r_{1,0}^{*}=r_{3}$ according to Lemma 2 , but in fact, $\hat{\rho}_{y}$ is closer to $r_{0}$ because of the asymmetry of the pseudo PAM. The proposed demapper uses a farther point that increases the absolute value of the LLR in (2). The increment of the absolute value of the LLR caused by the proposed demapper is bounded by

$$
\begin{aligned}
\Delta L & =\left(\left|\hat{\rho}_{y}-r_{3}\right|^{2}-\left|\hat{\rho}_{y}-r_{0}\right|^{2}\right) / N_{0} \\
& =\left(r_{3}-r_{0}\right)\left(r_{0}+r_{3}-2 \hat{\rho}_{y}\right) / N_{0} \\
& <\left(r_{3}-r_{0}\right)\left(r_{0}+r_{3}-r_{1}-r_{2}\right) / N_{0} .
\end{aligned}
$$

The exact and correct absolute LLR value is

$$
\begin{aligned}
\left|L_{1}\right| & =\left(\left|\hat{\rho}_{y}-r_{0}\right|^{2}-\left|\hat{\rho}_{y}-r_{2}\right|^{2}\right) / N_{0} \\
& =\left(r_{2}-r_{0}\right)\left(2 \hat{\rho}_{y}-r_{0}-r_{2}\right) / N_{0} \\
& >\left(r_{2}-r_{0}\right)\left(r_{1}-r_{0}\right) / N_{0} .
\end{aligned}
$$

Therefore, the ratio of $\Delta L$ over $\left|L_{1}\right|$ is bounded by

$$
\frac{\Delta L}{\left|L_{1}\right|}<\frac{\left(r_{3}-r_{0}\right)\left(r_{3}+r_{0}-r_{1}-r_{2}\right)}{\left(r_{2}-r_{0}\right)\left(r_{1}-r_{0}\right)} \approx 0.708 .
$$

The LLRs of the first bit of the pseudo 4PAM calculated by the Log-MAP, Max-Log-MAP, and our proposed demapper are shown in Fig. 5. The LLR calculated by our proposed demapper is exactly the same as that of the Max-Log-MAP demapper when $\hat{\rho}_{y}$ is outside the interval $\left(d_{1}, d_{2}\right)$. When $d_{1}<$ $\hat{\rho}_{y}<d_{2}$, the absolute value of the LLR calculated by our proposed demapper is slightly larger than that of the MaxLog-MAP demapper. It is interesting to note that the absolute value of the LLR calculated by the Log-MAP demapper is also slightly larger than that of the Max-Log-MAP demapper in some regions, and it is worth remembering that the Max-LogMAP solution itself is an approximation of the optimal LogMAP solution. 


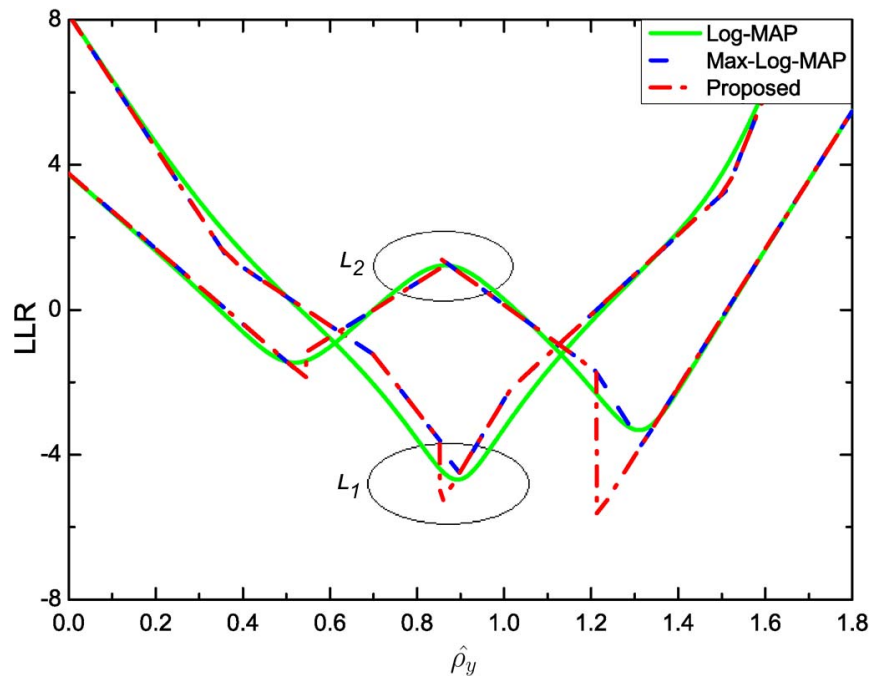

Fig. 6. LLRs of the first and second bits of the pseudo 8PAM decomposed from $(256=32 \times 8)$-APSK over the AWGN channel with $E_{s} / N_{0}=14 \mathrm{~dB}$.

The ratio (18) associated with the error is an upper bound. Furthermore, this error only exists when $\hat{\rho}_{y} \in\left(d_{1}, d_{2}\right)$, which does not frequently happen, as will be detailed later. Before analyzing the probability of $\hat{\rho}_{y}$ falling into an error interval, we further examine the larger constellation of $(256=32 \times 8)$ APSK.

b) $(256=32 \times 8)$-APSK: Similar to $(64=16 \times 4)$ APSK, for $(256=32 \times 8)$-APSK, the error also occurs when demapping the rightmost 3 bits, since we use the pseudo Gray 8PAM constellation. More specifically, if $\hat{\rho}_{y}$ is smaller than $\left(r_{3}+r_{4}\right) / 2$, the zeroth bit of $\mathbf{b}^{A^{*}}$ must be 0 . The constellation subset associated with the zeroth bit being 1 is $\mathcal{A}_{0}^{(1)}=\left\{r_{4}, r_{5}, r_{6}, r_{7}\right\}$, and obviously, the point closest to $\hat{\rho}_{y}$ in $\mathcal{A}_{0}^{(1)}$ is $r_{0,1}^{*}=r_{4}$, which is the same result as that given by Lemma 2. If $\hat{\rho}_{y}$ is larger than $\left(r_{3}+r_{4}\right) / 2$, we have $b_{0}^{A^{*}}=1$ and $r_{0,0}^{*}=r_{3}$, which is also identical to the result given by Lemma 2. Therefore, no error occurs when demapping the zeroth bit using Lemma 2. Demapping the first bit using Lemma 2 has one error interval $\left(\left(r_{3}+r_{4}\right) / 2,\left(r_{1}+r_{6}\right) / 2\right)$, whereas demapping the second bit using Lemma 2 has three error intervals $\left(\left(r_{0}+r_{3}\right) / 2, \quad\left(r_{1}+r_{2}\right) / 2\right), \quad\left(\left(r_{3}+r_{4}\right) / 2\right.$, $\left.\left(r_{2}+r_{5}\right) / 2\right)$, and $\left(\left(r_{5}+r_{6}\right) / 2,\left(r_{4}+r_{7}\right) / 2\right)$. The LLRs of the first and second bits related to the pseudo 8PAM calculated by the Log-MAP, Max-Log-MAP, and our proposed demapper are shown in Fig. 6. The LLR calculated by our proposed demapper is exactly the same as the Max-Log-MAP demapper when $\hat{\rho}_{y}$ is outside the error intervals. When $\hat{\rho}_{y}$ falls within one of the error intervals, the absolute value of the LLR calculated by our proposed demapper is slightly larger than that of the Max-Log-MAP demapper.

3) Error distribution: Since $\phi\left(x^{*}, y\right)$ represents the minimum phase distance between received signal $y$ and the constellation points, we have $\phi\left(x^{*}, y\right) \leq \pi / 2^{m_{1}}$. As the constellation order increases, $\phi\left(x^{*}, y\right)$ tends to 0 , and $\cos \left(\phi\left(x^{*}, y\right)\right)$ tends to 1 . For example, in the case of $(64=16 \times 4)$-APSK, we have $m_{1}=4, \phi\left(x^{*}, y\right) \leq \pi / 16=0.1963$, and $\cos \left(\phi\left(x^{*}, y\right)\right) \geq$ 0.9808 , whereas in the case of $(256=32 \times 8)$-APSK, we have $m_{1}=5, \phi\left(x^{*}, y\right) \leq \pi / 32=0.0982$, and $\cos \left(\phi\left(x^{*}, y\right)\right) \geq$
0.9952. Then, $\hat{\rho}_{y}$ can be approximated by $\rho_{y}$, which obeys a Rician distribution. Specifically

$$
p\left(\hat{\rho}_{y} \mid r\right) \approx \frac{2 \hat{\rho}_{y}}{N_{0}} \exp \left(-\frac{\hat{\rho}_{y}^{2}+r^{2}}{N_{0}}\right) I_{0}\left(\frac{2 r \hat{\rho}_{y}}{N_{0}}\right)
$$

where $r$ denotes the amplitude of transmitted signal $x$, and $I_{0}(\cdot)$ is the modified Bessel function of the first kind with order zero.

The error intervals for the $2^{m_{2}}$-ary pseudo PAM can be determined in the following recursive way.

i) For the zeroth bit and $m_{2} \geq 1$, there is no error interval.

ii) For the first bit and $m_{2}=2$, the error interval is $\left(\left(r_{1}+\right.\right.$ $\left.\left.r_{2}\right) / 2,\left(r_{0}+r_{3}\right) / 2\right)$.

iii) For the $k$ th bit, where $1 \leq k<m_{2}$ and $m_{2} \geq 2$, there are $2^{k}-1$ error intervals. We denote the $i$ th error interval as $\left(d_{i, 1}^{m_{2}, k}, d_{i, 2}^{m_{2}, k}\right)$, where

$d_{i, 1}^{m_{2}, k}=\min \left\{\left(r_{e_{i, 1}^{m_{2}, k}}+r_{e_{i, 2}^{m_{2}, k}}\right) / 2,\left(r_{e_{i, 3}^{m_{2}, k}}+r_{e_{i, 4}^{m_{2}, k}}\right) / 2\right\}$

$d_{i, 2}^{m_{2}, k}=\max \left\{\left(r_{e_{i, 1}^{m_{2}, k}}+r_{e_{i, 2}^{m_{2}, k}}\right) / 2,\left(r_{e_{i, 3}^{m_{2}, k}}+r_{e_{i, 4}^{m_{2}, k}}\right) / 2\right\}$

and $e_{i, j}^{m_{2}, k}$ denotes the index of the corresponding radius calculated by (12). For example, for case ii), we have $e_{1,1}^{2,1}=1, e_{1,2}^{2,1}=2, e_{1,3}^{2,1}=0$, and $e_{1,4}^{2,1}=3$. In general, index $e_{i, j}^{m_{2}, k}$ can be recursively determined from $e_{i, j}^{m_{2}-1, k-1}$ according to

$$
e_{i, j}^{m_{2}, k}= \begin{cases}e_{i, j}^{m_{2}-1, k-1}, & 1 \leq i \leq 2^{k-1}-1 \\ 2^{m_{2}}-1-e_{i, j}^{m_{2}-1, k-1}, & 1 \leq j \leq 4 \\ & 2^{k-1} \leq i<2^{k}-1 \\ 2^{m_{2}-1}-1, & 1 \leq j \leq 4 \\ 2^{m_{2}-1}, & i=2^{k}-1 ; j=1 \\ 2^{m_{2}-1}-2^{m_{2}-k-1}-1, & i=2^{k}-1 ; j=2 \\ 2^{m_{2}-1}+2^{m_{2}-k-1}, & i=2^{k}-1 ; j=3\end{cases}
$$

For the product-APSK constellation set $\mathcal{X}$, each ring has the same number of points, and radius $r$ is uniformly distributed over set $\mathcal{A}$. Therefore, the probability of $\hat{\rho}_{y}$ falling into the error interval $\left(d_{i, 1}^{m_{2}, k}, d_{i, 2}^{m_{2}, k}\right)$ is readily shown to be

$$
\begin{aligned}
P & \left(d_{i, 1}^{m_{2}, k}<\hat{\rho}_{y}<d_{i, 2}^{m_{2}, k}\right) \\
& =\sum_{s=0}^{2^{m_{2}-1}} P\left(r_{s}\right) P\left(d_{i, 1}^{m_{2}, k}<\hat{\rho}_{y}<d_{i, 2}^{m_{2}, k} \mid r_{s}\right) \\
& =\frac{1}{2^{m_{2}}} \sum_{s=0}^{2^{m_{2}-1}} \int_{d_{i, 1}^{m_{2}, k}}^{d_{i, 2}^{m_{2}, k}} p\left(\hat{\rho}_{y} \mid r_{s}\right) d \hat{\rho}_{y} .
\end{aligned}
$$

It is clear that (23) does not have a closed-form expression. Fortunately, since the Rician distribution can be approximated by the Gaussian distribution at a high SNR, we have

$$
\begin{aligned}
& P\left(d_{i, 1}^{m_{2}, k}<\hat{\rho}_{y}<d_{i, 2}^{m_{2}, k}\right) \\
& \quad \approx \frac{1}{2^{m_{2}}} \sum_{s=0}^{2^{m_{2}-1}}\left(Q\left(\frac{d_{i, 1}^{m_{2}, k}-r_{s}}{\sqrt{N_{0} / 2}}\right)-Q\left(\frac{d_{i, 2}^{m_{2}, k}-r_{s}}{\sqrt{N_{0} / 2}}\right)\right)
\end{aligned}
$$




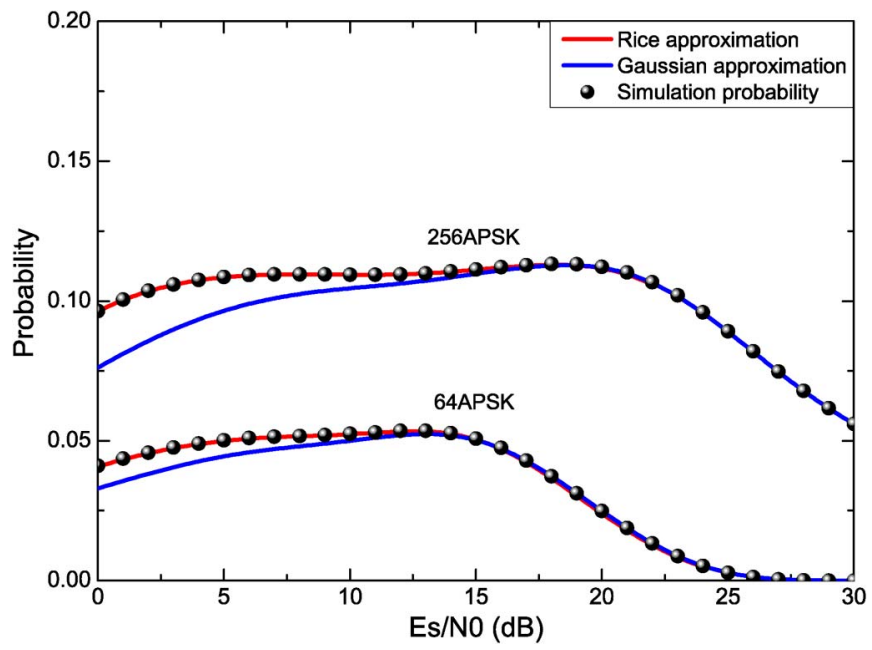

Fig. 7. Probability of $\hat{\rho}_{y}$ falling into the error interval(s) for $(64=16 \times 4)$ APSK and $(256=32 \times 8)$-APSK, for the AWGN channel.

and the probability of $\hat{\rho}_{y}$ falling into the error intervals can be obtained by

$$
\begin{aligned}
P_{e} \approx & \frac{1}{2^{m_{2}}} \\
& \times \sum_{s=0}^{2^{m_{2}-1}} \sum_{k=1}^{m_{2}-1} \sum_{i=1}^{2^{k}-1}\left(Q\left(\frac{d_{i, 1}^{m_{2}, k}-r_{s}}{\sqrt{N_{0} / 2}}\right)-Q\left(\frac{d_{i, 2}^{m_{2}, k}-r_{s}}{\sqrt{N_{0} / 2}}\right)\right)
\end{aligned}
$$

where $Q(x)=(1 / \sqrt{2 \pi}) \int_{x}^{\infty} \exp \left(-u^{2} / 2\right) d u$ represents the standard tail probability function of the Gaussian distribution with zero mean and unity variance.

For the case of $(64=16 \times 4)$-APSK, the probability of $\hat{\rho}_{y}$ falling into the error interval is shown in Fig. 7, as the function of the SNR $=E_{s} / N_{0}$ over the AWGN channel. Three $P_{e}$ 's are shown in Fig. 7, namely, the two theoretical $P_{e}$ 's derived by the Rician and Gaussian approximations and the probability $P_{e}$ obtained by simulation. It can be observed that the probability of $\hat{\rho}_{y}$ falling into the error interval is quite small even at low SNRs. At high SNRs, the Gaussian approximation matches well with the simulation result, and probability $P_{e}$ tends to zero with the increase in the SNR. This is due to the fact that received signal $y$ is likely to be very close to transmitted signal $x$ at a high SNR, and consequently, the probability of $\hat{\rho}_{y}$ falling into the error interval becomes extremely small.

Fig. 7 also shows the probability of $\hat{\rho}_{y}$ falling into the error intervals for $(256=32 \times 8)$-APSK for transmission over the AWGN channel at different SNR values. Probability $P_{e}$ is much higher than that of 64-APSK, since 256-APSK has more error intervals, but it is no more than $12 \%$ at low SNRs. At high SNRs, the Gaussian approximation matches well with the simulation result, and the probability decreases with the increase in the SNR. Probability $P_{e}$ tends to zero, given a sufficiently high SNR value, which is outside the SNR region shown in Fig. 7.

Our theoretical analysis of $(64=16 \times 4)$-APSK and $(256=$ $32 \times 8)$-APSK, therefore, shows that the error caused by the proposed simplified demapper is relatively small compared with the accurate LLR, and the probability of $\hat{\rho}_{y}$ falling into the error intervals is also small (less than $6 \%$ for 64-APSK and less than $12 \%$ for $256-$ APSK). Moreover, probability $P_{e}$ tends to zero at a sufficiently high SNR value. We can conclude that the performance degradation associated with the proposed demapper is negligible for $(64=16 \times 4)$-APSK and $(256=$ $32 \times 8)$-APSK, in comparison with that of the Max-Log-MAP demapper. This will be further demonstrated by the bit error rate (BER) simulation results in Section IV.

It should be noted that Lemma 2 and 3 can be implemented with the aid of a lookup table that defines the interval of $y$ and identifies which particular $k_{i}^{*}$ is used for each of the intervals specified by a set of thresholds. For nonuniform constellations such as product-APSK, we can use a larger lookup table, which contains the additional error intervals required for maintaining the performance, albeit this requires more comparison operations and an increased storage capacity.

\section{Simulation Results}

The BER performance of the proposed soft demapper was evaluated by simulation. According to our analysis presented in the previous sections, the proposed soft demapper achieves exactly the same performance as the standard Max-Log-MAP demapper for Gray-labeled PAM, PSK, and QAM. By contrast, it suffers from a slight performance loss for the Gray-labeled product-APSK because of the nonuniformly spaced pseudo PAM constellation embedded in the product-APSK. We therefore carried out simulations for the QAM and product-APSK constellations. The simulation parameters are listed as follows.

- Constellation Labeling: gray-labeled 64QAM, $(64=$ $16 \times 4)$-APSK, 256QAM and $(256=32 \times 8)$-APSK;

- Demapper: the standard Max-Log-MAP demapper and the proposed simplified soft demapper;

- Decoder: the 1/2-rate 64 800-bit long low-density paritycheck (LDPC) code of DVB-T2 was employed, whereby the normalized Min-Sum decoding algorithm with a normalization factor of $\alpha=1 / 0.875$ was selected [22]. The maximum number of LDPC iterations was set to 50;

- Channel: AWGN and independent identically distributed Rayleigh fading channels.

The achievable BER performance is shown in Figs. 8 and 9 for the AWGN and Rayleigh fading channels, respectively. It can be observed that the BER curves obtained by the MaxLog-MAP and our simplified demappers are overlapped for the Gray-labeled 64QAM and 256QAM over both the AWGN and Rayleigh fading channels. This confirms that the soft information calculated by our proposed demapper is exactly the same as that of the Max-Log-MAP demapper. The results shown in Figs. 8 and 9 also confirm that for the Gray-labeled product-APSK, the performance degradation caused by the proposed demapper is negligible compared with the Max-LogMAP demapper. Specifically, at the BER of $10^{-5}$, the performance loss is below $0.05 \mathrm{~dB}$ for the Gray-labeled 64APSK and 256APSK over both AWGN and Rayleigh channels, as shown in Figs. 8 and 9. As expected, the performance degradation in the case of $(256=32 \times 8)$-APSK is slightly higher than that of the $(64=16 \times 4)$-APSK, owing to the fact that $256 \mathrm{APSK}$ 


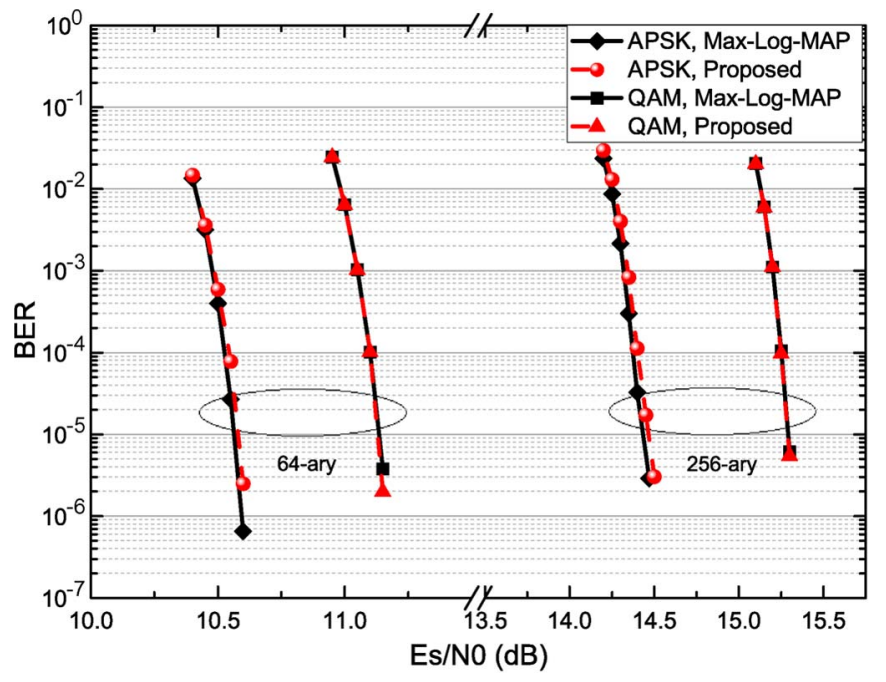

Fig. 8. BER performance comparison over the AWGN channel.

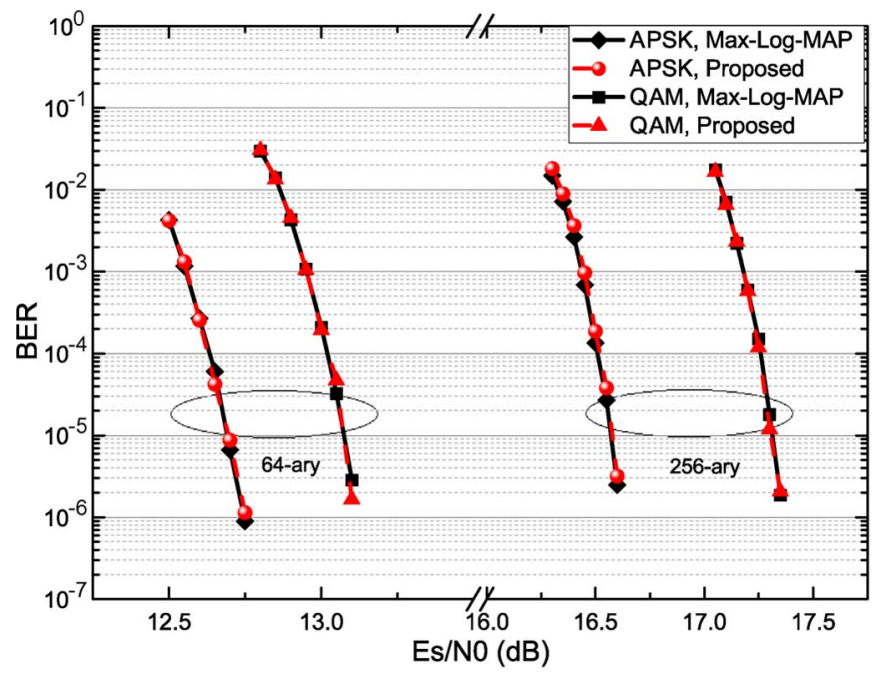

Fig. 9. BER performance comparison over the Rayleigh fading channel.

has one more bit related to the pseudo PAM. However, the performance loss still remains below $0.05 \mathrm{~dB}$ for 256APSK.

\section{CONCLUSION}

In this paper, a universal simplified soft demapper has been proposed for various binary-reflected Gray-labeled constellations. For the constellation of size $2^{m}$, our proposed demapper imposes a low-complexity order of $\mathrm{O}(m)$, instead of the complexity order of $\mathrm{O}\left(2^{m}\right)$ imposed by the standard Max-LogMAP demapper. Our theoretical analysis and simulation results have shown that the proposed simplified demapper achieves exactly the same performance as that of the Max-Log-MAP solution for Gray-labeled PAM, PSK, and QAM, whereas for the Gray-labeled product-APSK, the performance degradation caused by our simplified demapper remains negligible compared with that of the Max-Log-MAP demapper. More particularly, we have verified that this performance loss is less than $0.05 \mathrm{~dB}$ for both $(64=16 \times 4)$-APSK and $(256=32 \times 8)$ APSK for transmission over both the AWGN and Rayleigh fading channels.

\section{APPENDIX A}

PROOF OF LEMMA 2

Once $x^{*}$ and $\mathbf{b}^{*}$ are determined, constellation subset $\mathcal{X}_{i}^{\left(\overline{b_{i}^{*}}\right)}$ can be written as

$$
\mathcal{X}_{i}^{\left(\overline{b_{i}^{*}}\right)}=\left\{x_{k} \mid x_{k} \in \mathcal{X}, c_{i-1}^{k} \oplus c_{i}^{k}=\overline{b_{i}^{*}}\right\}
$$

where $\mathbf{c}^{k}=\left(c_{0}^{k} c_{1}^{k} \ldots c_{m-1}^{k}\right)$ denotes the binary representation of $k$, and we have $c_{-1}^{k}=0$. By denoting the nearest constellation point to $x^{*}$ in subset $\mathcal{X}_{i}^{\left(\overline{b_{i}^{*}}\right)}$ as the $k_{i}^{*}$ th constellation point $x_{k_{i}^{*}}$, we have

$$
\begin{aligned}
& x_{k_{i}^{*}}=\arg \min _{x \in \mathcal{X}_{i}^{\left(b_{i}^{*}\right)}}\left|x^{*}-x\right| \\
& k_{i}^{*}=\arg \min _{k \in \mathcal{K}_{i}^{\left(\bar{b}_{i}^{*}\right)}}\left|k^{*}-k\right|
\end{aligned}
$$

where $\mathcal{K}_{i}^{\left(\overline{b_{i}^{*}}\right)}=\left\{k \mid 0 \leq k<2^{m}, c_{i-1}^{k} \oplus c_{i}^{k}=\overline{b_{i}^{*}}\right\}$ denotes the index set corresponding to $\mathcal{X}_{i}^{\left(\overline{b_{i}^{*}}\right)}$.

For $k \in \mathcal{K}_{i}^{\left(\overline{b_{i}^{*}}\right)}$, we can express $k$ as $k=\sum_{j=0}^{m-1} c_{j}^{k} 2^{m-j-1}$, where we have $c_{i-1}^{k} \oplus c_{i}^{k}=\overline{b_{i}^{k^{*}}}=\overline{c_{i-1}^{k^{*}} \oplus c_{i}^{k^{*}}}$. Therefore, we have

$$
c_{i-1}^{k}=\overline{c_{i-1}^{k^{*}}} \text { and } c_{i}^{k}=c_{i}^{k^{*}} \text { or } c_{i-1}^{k}=c_{i-1}^{k^{*}} \text { and } c_{i}^{k}=\overline{c_{i}^{k^{*}}} .
$$

We now discuss the two situations.

i) The case of $c_{i-1}^{k}=\overline{c_{i-1}^{k^{*}}}$ and $c_{i}^{k}=c_{i}^{k^{*}}$. We have $c_{i-1}^{k^{*}}-$ $c_{i-1}^{k}= \pm 1$, and

$$
\begin{aligned}
& \left|\sum_{j_{1}=0}^{i-2}\left(c_{j_{1}}^{k^{*}}-c_{j_{1}}^{k}\right) 2^{m-j_{1}-1}+\left(c_{i-1}^{k^{*}}-c_{i-1}^{k}\right) 2^{m-i}\right| \\
& \quad=2^{m-i}\left|\sum_{j_{1}=0}^{i-2}\left(c_{j_{1}}^{k^{*}}-c_{j_{1}}^{k}\right) 2^{i-j_{1}-1}+\left(c_{i-1}^{k^{*}}-c_{i-1}^{k}\right)\right| \\
& \quad \geq 2^{m-i}
\end{aligned}
$$

where the inequality follows from the fact that $\sum_{j_{1}=0}^{i-2}\left(c_{j_{1}}^{k^{*}}-c_{j_{1}}^{k}\right) 2^{i-j_{1}-1}$ must be even and that $c_{i-1}^{k^{*}}-c_{i-1}^{k}$ is odd. We also have

$$
\begin{gathered}
\left|\sum_{j_{2}=i+1}^{m-1}\left(c_{j_{2}}^{k^{*}}-c_{j_{2}}^{k}\right) 2^{m-j_{2}-1}\right| \leq \sum_{j_{2}=i+1}^{m-1}\left|c_{j_{2}}^{k^{*}}-c_{j_{2}}^{k}\right| 2^{m-j_{2}-1} \\
\leq \sum_{j_{2}=i+1}^{m-1} 2^{m-j_{2}-1}=2^{m-i-1}-1
\end{gathered}
$$

Then, we can find the lower bound of $\left|k^{*}-k\right|$ as

$$
\begin{aligned}
\left|k^{*}-k\right|= & \mid \sum_{j_{1}=0}^{i-2}\left(c_{j_{1}}^{k^{*}}-c_{j_{1}}^{k}\right) 2^{m-j_{1}-1}+\left(c_{i-1}^{k^{*}}-c_{i-1}^{k}\right) 2^{m-i} \\
& \quad+\sum_{j_{2}=i+1}^{m-1}\left(c_{j_{2}}^{k^{*}}-c_{j_{2}}^{k}\right) 2^{m-j_{2}-1} \mid \\
\geq & \left|2^{m-i}-\left(2^{m-i-1}-1\right)\right|=2^{m-i-1}+1
\end{aligned}
$$


ii) The case of $c_{i-1}^{k}=c_{i-1}^{k^{*}}$ and $c_{i}^{k}=\overline{c_{i}^{k^{*}}}$. If $\exists j_{1} \in$ $\{0,1, \ldots, i-2\}$, which makes $c_{j_{1}}^{k} \neq c_{j_{1}}^{k^{*}}$, then we have

$$
\begin{aligned}
\left|k^{*}-k\right|= & \mid \sum_{j_{1}=0}^{i-2}\left(c_{j_{1}}^{k^{*}}-c_{j_{1}}^{k}\right) 2^{m-j_{1}-1} \\
& +\sum_{j_{2}=i}^{m-1}\left(c_{j_{2}}^{k^{*}}-c_{j_{2}}^{k}\right) 2^{m-j_{2}-1} \mid \\
\geq & || \sum_{j_{1}=0}^{i-2}\left(c_{j_{1}}^{k^{*}}-c_{j_{1}}^{k}\right) 2^{m-j_{1}-1} \mid \\
& -\left|\sum_{j_{2}=i}^{m-1}\left(c_{j_{2}}^{k^{*}}-c_{j_{2}}^{k}\right) 2^{m-j_{2}-1}\right| \mid \\
\geq & \left|2^{m-i+1}-\left(2^{m-i}-1\right)\right|=2^{m-i}+1 .
\end{aligned}
$$

On the other hand, if $c_{j_{1}}^{k}=c_{j_{1}}^{k^{*}}$ for $0 \leq j_{1} \leq i-2$, we have

$$
\begin{aligned}
\mid k^{*}- & k \mid \\
= & \left|\left(c_{i}^{k^{*}}-\overline{c_{i}^{k^{*}}}\right) 2^{m-i-1}+\sum_{j_{2}=i+1}^{m-1}\left(c_{j_{2}}^{k^{*}}-c_{j_{2}}^{k}\right) 2^{m-j_{2}-1}\right| \\
= & 2^{m-i-1}-(-1)^{c_{i}^{*}} \sum_{j_{2}=i+1}^{m-1} c_{j_{2}}^{k^{*}} 2^{m-j_{2}-1} \\
& +(-1)^{c_{i}^{k^{*}}} \sum_{j_{2}=i+1}^{m-1} c_{j_{2}}^{k} 2^{m-j_{2}-1} .
\end{aligned}
$$

Apparently, the minimum of (34) is smaller than $2^{m-i-1}$ and, thus, smaller than both the lower bounds given in (32) and (33). Since the first two items in (34) are fixed, minimizing $\mid k^{*}-$ $k \mid$ is equivalent to minimizing $(-1)^{c_{i}^{*}} \sum_{j_{2}=i+1}^{m-1} c_{j_{2}}^{k} 2^{m-j_{2}-1}$. Therefore, we have $c_{j}^{k_{i}^{*}}=c_{i}^{k^{*}}, i+1 \leq j \leq m-1$, and

$$
\begin{aligned}
k_{i}^{*} & =\sum_{j_{1}=0}^{i-2} c_{j_{1}}^{k^{*}} 2^{m-j_{1}-1}+\overline{c_{i}^{k^{*}}} 2^{m-i-1}+\sum_{j_{2}=i+1}^{m-1} c_{i}^{k^{*}} 2^{m-j_{2}-1} \\
& =2^{m-i-1}-c_{i}^{k^{*}}+\sum_{j=0}^{i-1} c_{j}^{k^{*}} 2^{m-j-1} .
\end{aligned}
$$

It is clear that $k_{i}^{*}$ is the unique solution of (28). Hence, $\forall k \in$ $\mathcal{K}_{i}^{\left(\overline{b_{i}^{*}}\right)} \backslash\left\{k_{i}^{*}\right\}$, we have $\left|k^{*}-k\right| \geq\left|k^{*}-k_{i}^{*}\right|+1$, and

$$
\left|x^{*}-x_{k}\right| \geq\left|x^{*}-x_{k_{i}^{*}}\right|+\delta .
$$

Since $x^{*}$ is the nearest constellation point to $y$, we obtain

$$
\left|y-h x^{*}\right| \leq|h| \delta / 2
$$

for $y \in\left[-2^{m-1}|h| \delta, 2^{m-1}|h| \delta\right]$. In this case, for $k \in \mathcal{K}_{i}^{\left(\overline{b_{i}^{*}}\right)}$ $\left\{k_{i}^{*}\right\}$, we have

$$
\begin{aligned}
\left|y-h x_{k}\right| & \geq\left|h\left(x^{*}-x_{k}\right)\right|-\left|y-h x^{*}\right| \\
& \geq|h|\left(\left|x^{*}-x_{k_{i}^{*}}\right|+\delta\right)-|h| \delta / 2 \\
& \geq\left|h\left(x^{*}-x_{k_{i}^{*}}\right)\right|+\left|y-h x^{*}\right| \geq\left|y-h x_{k_{i}^{*}}\right| .
\end{aligned}
$$

It is easy to find that this inequality still holds when $y$ is outside the interval $\left[-2^{m-1}|h| \delta, 2^{m-1}|h| \delta\right]$. Therefore, $x_{k_{i}^{*}}$ is not only the nearest constellation point to $x^{*}$ in $\mathcal{X}_{i}^{\left(\overline{b_{i}^{*}}\right)}$ but the nearest constellation point to $y$ in $\mathcal{X}_{i}^{\left(b_{i}^{*}\right)}$ as well. This completes the proof of Lemma 2.

\section{APPENDIX B}

\section{PROOF OF THE TRIANGLE INEQUALITY of the Phase Distance}

From (8), $\phi(x, y)$ can be rewritten as $\phi(x, y)=\min \left\{\mid \varphi_{x}-\right.$ $\left.\varphi_{y}|, 2 \pi-| \varphi_{x}-\varphi_{y} \mid\right\}$. The proof is divided into three parts according to the values of $\left|\varphi_{x}-\varphi_{y}\right|$ and $\left|\varphi_{y}-\varphi_{z}\right|$.

i) If $\left|\varphi_{x}-\varphi_{y}\right| \leq \pi$ and $\left|\varphi_{y}-\varphi_{z}\right| \leq \pi$, we have

$\phi(x, y)+\phi(y, z)=\left|\varphi_{x}-\varphi_{y}\right|+\left|\varphi_{y}-\varphi_{z}\right| \geq\left|\varphi_{x}-\varphi_{z}\right| \geq \phi(x, z)$.

ii) For $\left|\varphi_{x}-\varphi_{y}\right|>\pi$ and $\left|\varphi_{y}-\varphi_{z}\right| \leq \pi$ or $\left|\varphi_{x}-\varphi_{y}\right| \leq \pi$ and $\left|\varphi_{y}-\varphi_{z}\right|>\pi$, without loss of generality, we assume $\left|\varphi_{x}-\varphi_{y}\right|>\pi$ and $\left|\varphi_{y}-\varphi_{z}\right| \leq \pi$. Then, we have

$$
\begin{aligned}
\phi(x, y)+\phi(y, z) & =2 \pi-\left|\varphi_{x}-\varphi_{y}\right|+\left|\varphi_{y}-\varphi_{z}\right| \\
& \geq 2 \pi-\left|\varphi_{x}-\varphi_{z}\right| \geq \phi(x, z) .
\end{aligned}
$$

iii) For $\left|\varphi_{x}-\varphi_{y}\right|>\pi$ and $\left|\varphi_{y}-\varphi_{z}\right|>\pi$, without loss of generality, we assume $\varphi_{x} \geq \varphi_{z}$. Since $\varphi_{x}, \varphi_{y}$, and $\varphi_{z}$ are all inside the interval $[0,2 \pi]$, we have $\varphi_{x} \geq \varphi_{z}>\varphi_{y}+\pi$ or $\varphi_{z} \leq \varphi_{x}<\varphi_{y}-\pi$. If $\varphi_{x} \geq \varphi_{z}>\varphi_{y}+\pi$, we have

$$
\left|\varphi_{x}-\varphi_{y}\right|+\left|\varphi_{y}-\varphi_{z}\right|+\left|\varphi_{x}-\varphi_{z}\right|=2 \varphi_{x}-2 \varphi_{y}<4 \pi \text {. }
$$

If $\varphi_{z} \leq \varphi_{x}<\varphi_{y}-\pi$, we have

$$
\left|\varphi_{x}-\varphi_{y}\right|+\left|\varphi_{y}-\varphi_{z}\right|+\left|\varphi_{x}-\varphi_{z}\right|=2 \varphi_{y}-2 \varphi_{z}<4 \pi .
$$

In both cases, we have

$$
\begin{aligned}
& \phi(x, y)+\phi(y, z) \\
& \quad=2 \pi-\left|\varphi_{x}-\varphi_{y}\right|+2 \pi-\left|\varphi_{y}-\varphi_{z}\right|>\left|\varphi_{x}-\varphi_{z}\right| \\
& \quad \geq \phi(x, z) .
\end{aligned}
$$

This completes the proof.

\section{APPENDIX C}

\section{PROOF OF LEMMA 3}

The definitions of $\mathcal{X}_{i}^{\left(\overline{b_{i}^{*}}\right)}$ and $x_{k_{i}^{*}}$ are the same as given in (26) and (27). Noting that

$$
\begin{aligned}
\left|x^{*}-x\right|^{2} & =\left|\sqrt{E_{s}} \exp \left(\mathrm{j} \varphi_{x^{*}}\right)-\sqrt{E_{s}} \exp \left(\mathrm{j} \varphi_{x}\right)\right|^{2} \\
& =2 E_{s}-2 E_{s} \cos \left(\phi\left(x^{*}, x\right)\right)
\end{aligned}
$$


we have

$$
k_{i}^{*}=\arg \min _{k \in \mathcal{K}_{i}^{\left(b_{i}^{*}\right)}} \phi\left(x_{k^{*}}, x_{k}\right) .
$$

Similar to the proof of Lemma 2, we can get the unique solution of $k_{i}^{*}$ as shown in (11), which means that $\forall k \in \mathcal{K}_{i}^{\left(\overline{b_{i}^{*}}\right)} \backslash\left\{k_{i}^{*}\right\}$, we have

$$
\phi\left(x_{k}, x^{*}\right) \geq \phi\left(x^{*}, x_{k_{i}^{*}}\right)+2 \pi / 2^{m} .
$$

Since $x^{*}$ is the nearest constellation point to $y$, we obtain

$$
\phi\left(x^{*}, y\right) \leq \pi / 2^{m}
$$

According to (7), (9), (46), and (47), we have, $\forall k \in \mathcal{K}_{i}^{\left(\overline{b_{i}^{*}}\right)} \backslash\left\{k_{i}^{*}\right\}$

$$
\begin{aligned}
\phi\left(x_{k}, y\right) & \geq \phi\left(x^{*}, x_{k}\right)-\phi\left(x^{*}, y\right) \\
& \geq \phi\left(x^{*}, x_{k_{i}^{*}}\right)+2 \pi / 2^{m}-\pi / 2^{m} \\
& \geq \phi\left(x^{*}, x_{k_{i}^{*}}\right)+\phi\left(x^{*}, y\right) \geq \phi\left(x_{k_{i}^{*}}, y\right) \\
\left|y-h x_{k}\right| & \geq\left|y-h x_{k_{i}^{*}}\right| .
\end{aligned}
$$

Therefore, $x_{k_{i}^{*}}$ is not only the nearest constellation point to $x^{*}$ in $\mathcal{X}_{i}^{\left(\overline{b_{i}^{*}}\right)}$ but the nearest constellation point to $y$ in $\mathcal{X}_{i}^{\left(\overline{b_{i}^{*}}\right)}$ as well. This completes the proof.

\section{REFERENCES}

[1] Digital Video Broadcasting (DVB); Frame Structure Channel Coding and Modulation for a Second Generation Digital Terrestrial Television Broadcasting System (DVB-T2), ETSI EN Std. 302755 V1.3.1, Apr. 2012.

[2] Digital Video Broadcasting (DVB); Frame Structure Channel Coding and Modulation for a Second Generation Digital Transmission System for Cable Systems (DVB-C2), ETSI EN Std. 302769 V1.2.1, Apr. 2012.

[3] Third-Generation Partnership Project (3GPP); Technical specification group radio access network; Physical layer aspects for evolved UTRA, Third-Generation Partnership Project (3GPP), Sophia Antipolis, France. [Online]. Available: http://www.3gpp.org/ftp/Specs/html-info/25814.htm

[4] J. Erfanian, S. Pasupathy, and G. Gulak, "Reduced complexity symbol detectors with parallel structures for ISI channels," IEEE Trans. Commun., vol. 42, no. 2/3/4, pp. 1661-1671, Feb./Mar./Apr. 1994.

[5] P. Robertson, E. Villebrun, and P. Hoeher, "A comparison of optimal and sub-optimal MAP decoding algorithms operating in the log domain," in Proc. IEEE ICC, Seattle, WA, USA, Jun. 18-22, 1995, vol. 2, pp. 1009-1013.

[6] L. Wang, D. Xu, and X. Zhang, "Recursive bit metric generation for PSK signals with Gray labeling," IEEE Commun. Lett., vol. 16, no. 2, pp. 180182, Feb. 2012.

[7] E. Akay and E. Ayanoglu, "Low complexity decoding of bit-interleaved coded modulation for M-ary QAM," in Proc. IEEE ICC, Paris, France, Jun. 20-24, 2004, vol. 2, pp. 901-905.

[8] C.-W. Chang, P.-N. Chen, and Y. S. Han, "A systematic bit-wise decomposition of M-ary symbol metric," IEEE Trans. Wireless Commun., vol. 5, no. 10 , pp. $2742-2751$, Oct. 2006

[9] F. Tosato and P. Bisaglia, "Simplified soft-output demapper for binary interleaved COFDM with application to HIPERLAN/2," in Proc. IEEE ICC, New York, NY, USA, Apr. 28/May 2, 2002, vol. 2, pp. 664-668.

[10] M. Zhang and S. Kim, "Efficient soft demapping for M-ary APSK," in Proc. ICTC, Seoul, Korea, Sep. 28-30, 2011, pp. 641-644.

[11] G. Gül, A. Vargas, W. H. Gerstacker, and M. Breiling, "Low complexity demapping algorithms for multilevel codes," IEEE Trans. Commun., vol. 59, no. 4, pp. 998-1008, Apr. 2011.

[12] J. W. Park, M. H. Sunwoo, P. S. Kim, and D.-I. Chang, "Low complexity soft-decision demapper for high order modulation of DVB-S2 system," in Proc. ISOCC, Busan, Korea, Nov. 24, 2008, pp. II-37-II-40.

[13] D. Pérez-Calderoñ, V. Baena-Lecuyer, A. C. Oria, P. López, and J. G. Doblado, "Rotated constellation demapper for DVB-T2," Electron. Lett., vol. 47, no. 1, pp. 31-32, Jan. 2011.

[14] S. Tomasin and M. Butussi, "Low complexity demapping of rotated and cyclic Q delayed constellations for DVB-T2," IEEE Wireless Commun. Lett., vol. 1, no. 2, pp. 81-84, Apr. 2012.
[15] Y. Fan and C. Tsui, "Low-complexity rotated QAM demapper for the iterative receiver targeting DVB-T2 standard," in Proc. IEEE VTC-Fall, Québec City, QC, Canada, Sep. 3-6, 2012, pp. 1-5.

[16] Z. Liu, Q. Xie, K. Peng, and Z. Yang, "APSK constellation with Gray mapping," IEEE Commun. Lett., vol. 15, no. 12, pp. 1271-1273, Dec. 2011.

[17] Q. Xie, Z. Wang, and Z. Yang, "Simplified soft demapper for APSK with product constellation labeling," IEEE Trans. Wireless Commun., vol. 11, no. 7, pp. 2649-2657, Jul. 2012.

[18] F. Gray, "Pulse code communications," US Patent 2632 058, Mar. 17, 1953

[19] E. Agrell, J. Lassing, E. G. Ström, and T. Ottosson, "On the optimality of the binary reflected Gray code," IEEE Trans. Inf. Theory, vol. 50, no. 12, pp. 3170-3182, Dec. 2004.

[20] E. M. Reingold, J. Nievergelt, and N. Deo, Combinatorial Algorithms: Theory and Practice. Englewood Cliffs, NJ, USA: Prentice-Hall, 1977.

[21] R. De Gaudenzi, A. Guillen, and A. Martinez, "Performance analysis of turbo-coded APSK modulations over nonlinear satellite channels," IEEE Trans. Wireless Commun., vol. 5, no. 9, pp. 2396-2407, Sep. 2006.

[22] J. Chen and M. P. C. Fossorier, "Near optimum universal belief propagation based decoding of low-density parity check codes," IEEE Trans. Commun., vol. 50, no. 3, pp. 406-414, Mar. 2002.

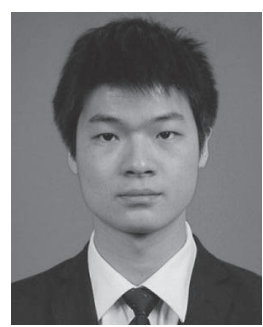

Qi Wang received the B.S. degree from Tsinghua University, Beijing, China, in 2011, where he is currently working toward the Ph.D. degree with the Department of Electronic Engineering.

His current research interests include optical wireless communications and channel coding and modulation.

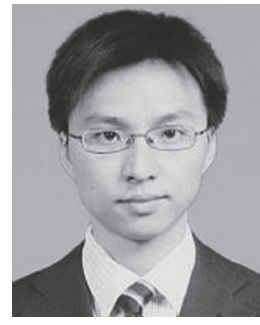

Qiuliang Xie received the B.Eng. degree in telecommunication engineering from Beijing University of Posts and Telecommunications, Beijing, China, in 2006 and the Ph.D. degree in electronic engineering from Tsinghua University, Beijing, in 2011, both with high honors.

From July 2011 to March 2013, he was with Digital TV National Engineering Laboratory (Beijing) Co., Ltd., where he participated in developing China's next-generation broadcasting standard. He is currently a Postdoctoral Fellow with the Department of Radiation Oncology, University of California, Los Angeles, CA, USA, where he is engaged in medical image processing. His main research interests include medical image processing and broadband wireless communication, specifically including information theory, coding theory, and image/signal processing theories.

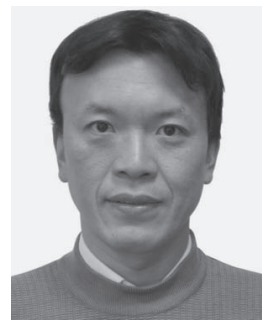

Zhaocheng Wang (SM'10) received the B.S., M.S., and Ph.D. degrees from Tsinghua University, Beijing, China, in 1991, 1993, and 1996, respectively.

From 1996 to 1997, he was a Postdoctoral Fellow with Nanyang Technological University, Singapore. From 1997 to 1999, he was with OKI Techno Centre (Singapore) Pte. Ltd., first as a Research Engineer and then as a Senior Engineer. From 1999 to 2009, he was with Sony Deutschland $\mathrm{GmbH}$, first as a Senior Engineer and then as a Principal Engineer. $\mathrm{He}$ is currently a Professor with the Department of Electronic Engineering, Tsinghua University. He has published over 80 technical papers. He is the holder of 30 U.S./European Union patents. His research interests include wireless communications, digital broadcasting, and millimeter-wave communications.

Dr. Wang has served as a Technical Program Committee Cochair/Member of many international conferences. He is a Fellow of the Institution of Engineering and Technology. 


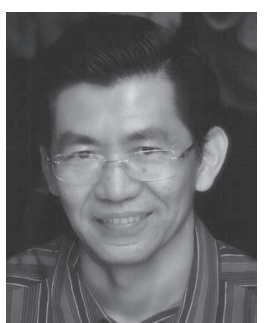

Sheng Chen (M'90-SM'97-F'08) received the B.Eng. degree in control engineering from East China Petroleum Institute, Dongying, China, in 1982; the Ph.D. degree in control engineering from City University London, London, U.K., in 1986; and the D.Sc. degree from the University of Southampton, Southampton, U.K., in 2005.

From 1986 to 1999, he held research and academic appointments with The University of Sheffield, The University of Edinburgh, and the University of Portsmouth, all in the U.K. Since 1999, he has been with Electronics and Computer Science, University of Southampton, where he is currently a Professor of intelligent systems and signal processing. $\mathrm{He}$ is a Distinguished Adjunct Professor with King Abdulaziz University, Jeddah, Saudi Arabia. He has published over 480 research papers. His recent research interests include adaptive signal processing, wireless communications, modeling and identification of nonlinear systems, neural network and machine learning, intelligent control system design, evolutionary computation methods, and optimization.

Dr. Chen is a Chartered Engineer and a Fellow of the Institution of Engineering and Technology. He was an Institute for Scientific Information highly cited researcher in the engineering category in March 2004.

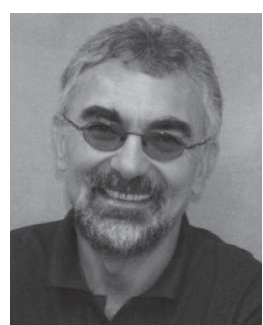

Lajos Hanzo (M'91-SM'92-F'04) received the M.S. degree (with first-class honors) in electronics and the Ph.D. degree from the Technical University of Budapest, Budapest, Hungary, in 1976 and 1983, respectively, the D.Sc. degree from the University of Southampton, Southampton, U.K., in 2004, and the "Doctor Honoris Causa" degree from the Technical University of Budapest in 2009.

During his 35-year career in telecommunications, he has held various research and academic posts in Hungary, Germany, and the U.K. Since 1986, he has been with the School of Electronics and Computer Science, University of Southampton, Southampton, U.K., where he holds the Chair for Telecommunications. Since 2009, he has been a Chaired Professor with Tsinghua University, Beijing China. He is currently directing a 100-strong academic research team, working on a range of research projects in the field of wireless multimedia communications sponsored by industry; the Engineering and Physical Sciences Research Council, U.K.; the European IST Programme; and the Mobile Virtual Centre of Excellence, U.K. He is an enthusiastic supporter of industrial and academic liaison and offers a range of industrial courses. He has successfully supervised 80 Ph.D. students, coauthored 20 John Wiley/IEEE Press books on mobile radio communications totaling in excess of 10000 pages, published more than 1250 research entries on IEEE Xplore, and presented keynote lectures. (For further information on research in progress and associated publications, please refer to http://www-mobile.ecs.soton.ac.uk.)

Dr. Hanzo is Fellow of the Royal Academy of Engineering, U.K., a Fellow of the Institution of Electrical Engineers, and a Governor of the IEEE Vehicular Technology Society. He has been a Technical Program Committee Chair and a General Chair for IEEE conferences. During 2008-2012, he was the Editor-inChief of the IEEE Press. He has received a number of distinctions. 